\title{
A mastication mechanism designed for testing temporomandibular joint implants
}

\author{
Ryan J. Frayne ${ }^{\mathrm{a}, *}$, Marvin Schwartz ${ }^{\mathrm{b}}$ and James P. Dickey ${ }^{\mathrm{a}}$ \\ ${ }^{a}$ School of Kinesiology/Faculty of Health Sciences, University of Western Ontario, London, ON, Canada \\ b4iBio, Pickering, ON, Canada
}

\begin{abstract}
The development of temporomandibular joint implants has involved simplified mechanical tests that apply pure vertical forces or pure rotational movements to the implant. The aim of this study was to develop a biological based mastication mechanism and conduct preliminary testing of a novel temporomandibular joint implant. The mechanism was designed to mimic temporomandibular joint loads by performing compression and anterior/posterior translation. Pilot testing was performed on six implant/joint specimens for seven consecutive hours, completing approximately 22,000 cycles at a frequency of approximately $1 \mathrm{~Hz}$. Each cycle had a joint compression phase $(67.3 \mathrm{~N}$ over $0.15 \mathrm{~s})$ followed by a translation phase $(8.67 \mathrm{~N}$ over $0.43 \mathrm{~s})$ that was similar to joint loads/motions that have been reported in vivo. This new mastication mechanism incorporates both anatomical and mechanical variability. The use of biological specimens is an important approach that can help bridge the gap between traditional synthetic implant materials/mechanical testing and in vivo testing.
\end{abstract}

Keywords: Implant dentistry, mastication, temporomandibular disorders (TMD), mechanical testing, biological specimen testing

\section{Introduction}

\subsection{Background of disease}

Temporomandibular joint disorders (TMD) cause a wide range of symptoms. Minor disorders are associated with symptoms of jaw clicking and minor pain [23], while more severe disorders are characterized by permanent disc displacement, interference in jaw opening, myofacial pain, headaches, jaw locking and degenerative joint disease [28, 31]. Surveys have reported that $20-45 \%$ of a general population can be affected by some degree of TMD [7, 11, 19]. Few [2 of 19 patients at 1 month and 7 of 19 patients at 6 months post Hyaluronic acid injection; 13] patients fail

*Corresponding author: Ryan J. Frayne, MSc., Joint Biomechanics Laboratory, School of Kinesiology, Thames Hall 2141, 1151 Richmond Street, The University of Western Ontario, London, ON, N6A 3K7 Canada. Tel.: +1 5196612111 ext. 88542; E-mail: rfrayne2@uwo.ca. to respond to conservative/minimally invasive treatments; however, those individuals that don't respond, require a more invasive treatment strategy [32]. In the most advanced cases of TMD (ankylosis, severe arthritic changes, tumors etc), autogenous implants and custom or predesigned partial or total alloplastic implants are the surgical alternatives for these individuals [12, 21, 26].

\subsection{Background on implants}

Currently there is no universally accepted implant for TMJ replacement [9], and accordingly new implants are being developed. Past implant failures can be attributed to a lack of a sound scientific approach and inadequate basic research to study the causes of damage [21]. Most TMJ prosthetic solutions, to date, have attempted to follow the orthopedic model of replacement and use man-made materials to replace damaged tissues. The future direction of joint repair 
is toward joint protection in the form of synthetic meniscal replacements (e.g. alloplastic implants) and bioengineered solutions that use cell growth to replace natural tissues (e.g. autogenous implants). There are positive and negatives to each surgical strategy. An advantage of tissue-engineered implants over synthetic implants is that natural tissues are more likely to be compatible with the existing tissues [29]. Tissueengineered implants have to be implemented at an earlier stage of disease in order to be effective; while, synthetic implants can be used to treat extensively damaged TMJs [29]. Both styles of implant require more research and in order to test them a more sophisticated testing model (involving biological tissues) needs to be developed that can assess the wear and durability of these new replacement modalities.

\subsection{Mechanical testing models}

It is difficult to reproduce the exact movements of the TMJ because of its complex nature. Most mechanical tests have been limited to friction loads being applied to the implants about a fixed axis [10, 24, 33]. There have also been some more advanced mechanisms [27]; however, the load timing during the chewing cycle is unclear. The difficulty in physically reproducing TMJ movements has led to the use of finite element models.

\subsection{Finite element models}

These models are capable of analyzing repetitive complex movements. They have been used to test the stress distribution of the Christensen Implant [20], and the TMJ effects of a tissue-engineered articular disc implant [1]. The later study was validated using a custom designed TMJ force applicator; however, along with other mechanical tests, the applicator was simplified to subjecting a vertical force to the symphysis and both implants [2]. Finite element models have been successfully employed for analyzing and predicting stress fractures [20], as well as predicting service life [6].

\subsection{Purpose}

The aim of this study was to develop an in vitro mastication mechanism, using biological tissues that can test novel synthetic implants and reproduce the physiological movements and forces of the TMJ.

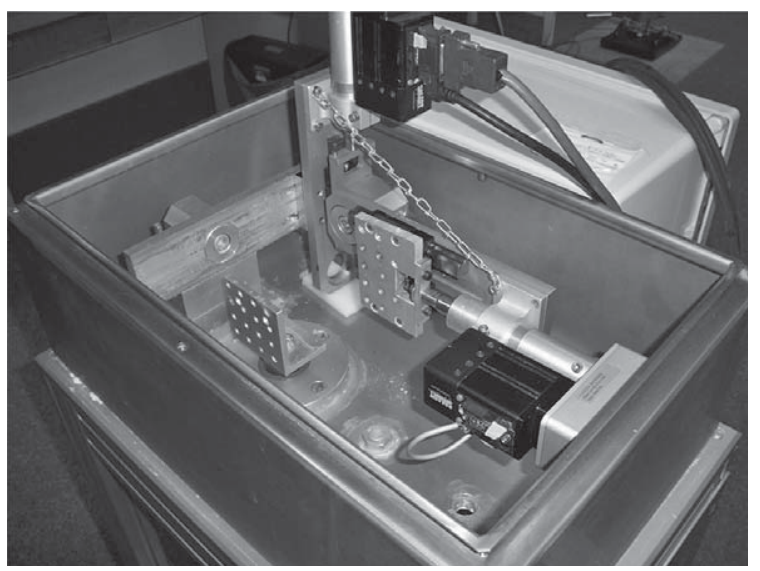

Fig. 1. The mastication mechanism within the fluid basin. The horizontal linear actuator (right) and the compression piston (coming through the bottom of the basin) are attached to the temporal bone and the mandible, respectably.

\section{Materials and methods}

\subsection{Mastication mechanism}

The custom-designed mastication mechanism replicated the two most prevalent movements in chewing, protrusion/retrusion and elevation/depression (compression). Protrusion/retrusion was accomplished using an electrical linear actuator (Fig. 1; Smart Motor, Ultra Motion, Cutchogue, NY, USA) that was capable of a total displacement of $50 \mathrm{~mm}$. This movement simulated the translation of the condylar-disc complex along the articular fossa and eminence. There were two linear actuators; one that controlled the horizontal translations of each stroke and another that controlled the vertical placement of the superior portion of the TMJ.

Compression is the principal form of loading in the TMJ [17, 30]. The mastication mechanism used two (air compression) parallel fluidic muscles (Fig. 2; MXAM-40-AA, Festo inc., Mississauga, Ontario, Canada) that lifted a piston upwards to contact the articular fossa. This movement represented the loading during the elevation/occlusal phase of the chewing cycle. The maximum force capacity of the muscles was 6000 N. A 27 gallon air compressor was used to supply air compression to the fluidic muscles. Quickexhaust valves (SE-3/8-B, Festo Inc., Mississauga, Ontario, Canada) at the base of the fluidic muscles increased the pneumatic discharge flow rate and effectively increased the frequency of the chewing cycles. 


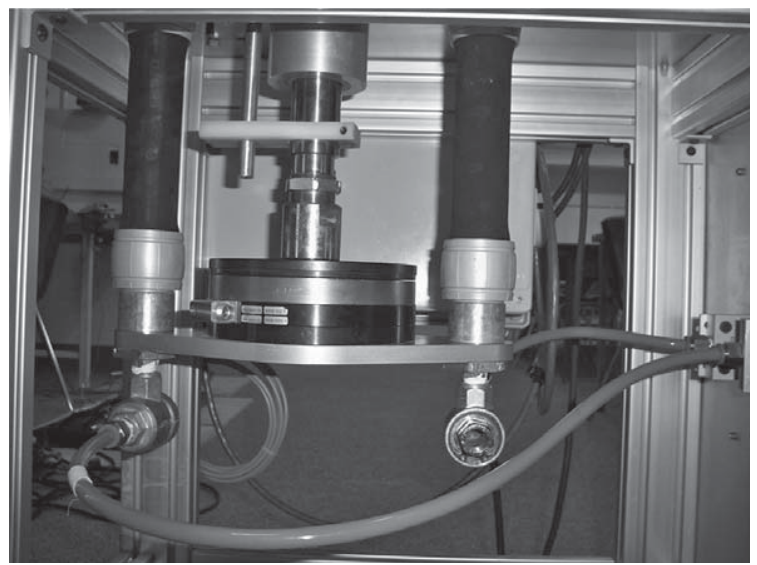

Fig. 2. The compression apparatus of the mastication mechanism. Two fluidic muscles are on either side of a pneumatic piston. The piston passes through a bushing at the bottom of the basin. Attached below the piston is a 6 degree load cell.

In order to minimize applied moments, the center of the TMJ joint (articular fossa) was placed in line with the horizontal linear actuator axis and the vertical actuator axis. A six-axis load cell (ATI Omega 160, Apex, $\mathrm{NC}$, USA) was positioned in series with the piston to measure the forces acting on the mandibular condyle (Fig. 2).

The movement of the translational linear actuator was controlled by a programmable logic controller (PLC; Micro Logix 1100, Rockwell Automation, Inc., Milwaukee, Wisconsin, United States of America). The PLC also controlled the timing and sequencing of the air compression valves and pauses between the protrusion/retrusion and elevation/depression movements.

The TMJs were attached to the mechanism via threaded aluminum plates that attached to the linear actuator and the compression piston. The temporal bone was attached to the mobile end of the linear actuator by bolting through the zygomatic arch (Fig. 3). The mandible attachment was similar to the temporal attachment, but used an L-shaped aluminum plate that butted to the end of the compression piston (Fig. 1).

The specimen was kept moist throughout testing by using a saline solution bath (Dulbecco's phosphate buffered saline without calcium or magnesium, MP Biomedicals, LLC, Solon, Ohio, USA). The solution was constrained around the specimen by a sac made from a piece of pure gum rubber $(60 \mathrm{~cm}$ by $60 \mathrm{~cm}$ and $3.2 \mathrm{~mm}$ thick). The laxity in the sac enabled motion of the jig without interference.

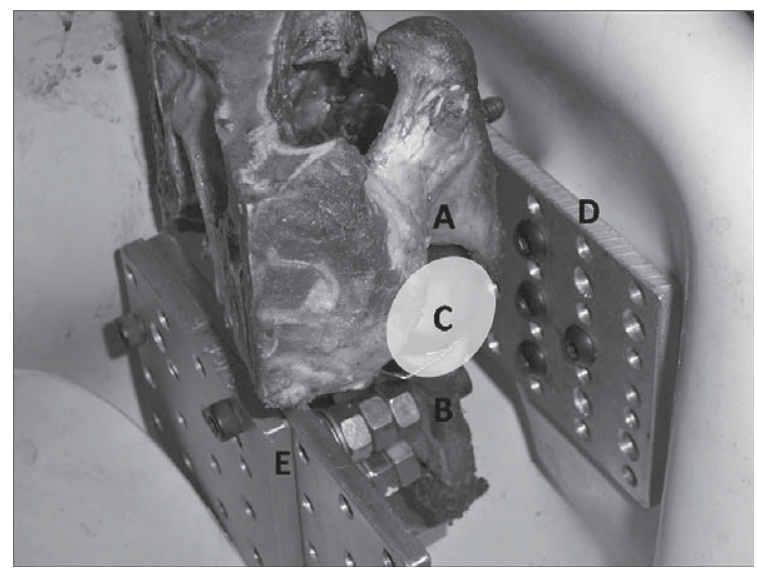

Fig. 3. A swine specimen sitting within the mechanism. (A) The temporal portion of the skull, with the temporal fossa located directly below the "A". (B) The mandible with the mandibular condyle located at "C". (C) The location of the implant. (D) The bracket that attaches to the temporal portion of the skull through the zygomatic arch. The active end of the horizontal linear actuator (Fig. 1) attaches to the other side of this bracket. (E) This bracket attaches to the mandible and was fastened on top of the compression piston (Fig. 1). Surrounding the attachment plates and specimen is the pure gum rubber sac that confined the saline bath during testing.

\subsection{Study design}

In this experiment swine TMJ specimens were used because of their similarities to human joints $[8,15]$. The swine TMJs were extracted from heads obtained from the University of Guelph Abattoir (Guelph, Ontario, Canada). We used fully mature specimens to ensure complete development of the temporomandibular bones.

The mastication mechanism applied a chewing frequency of approximately $1 \mathrm{~Hz}$. The mechanism ran for seven continuous hours resulting in 21,672 loading cycles, representing approximately two months of clinical use [27]. A translational distance of $5 \mathrm{~mm}$ and a compression force of approximately $60-70 \mathrm{~N}$ were used to mimic swine masticatory biomechanics based on predictions for a detailed analytical model of porcine chewing [22]. This model incorporated representations of six bilateral pairs of muscles activated using recorded electromyographic amplitudes and patterns from in vivo pig chewing.

In order to assess the mechanism's variability, the following data were collected and calculated: the average compression force and time, the average translational force and time, and the average mastication cycle frequency. 


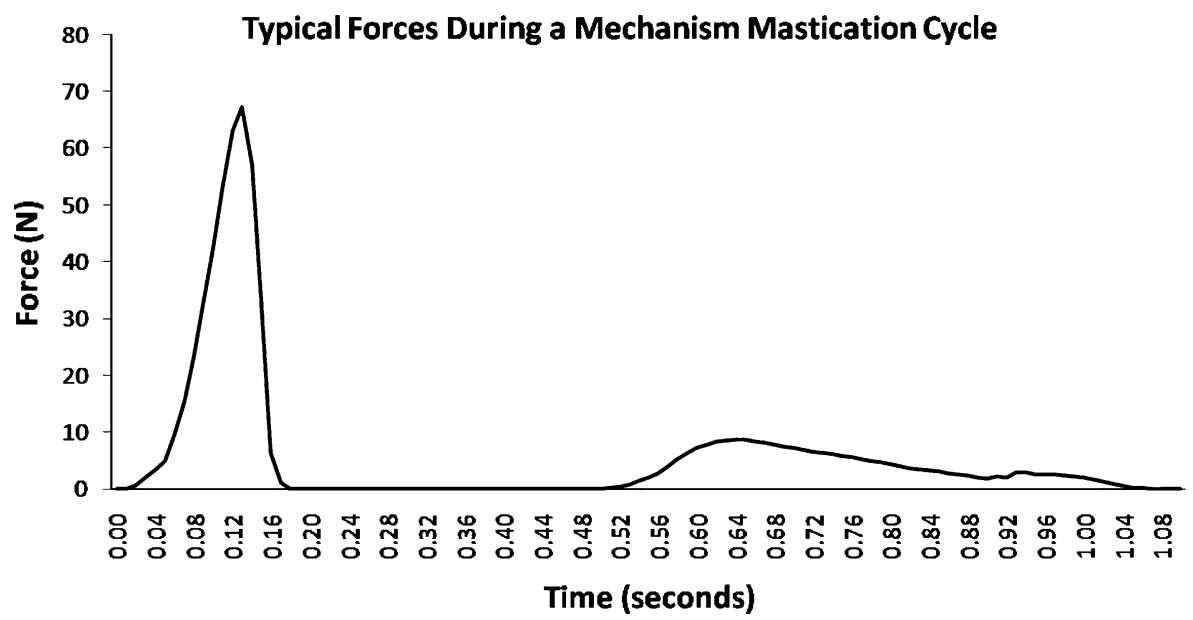

Fig. 4. Schematic of the mastication mechanism's chewing cycle. Zero force signifies no contact between the implant and the articular eminence/fossa. The prolonged loading following the translational phase is due to forces from the condyle sliding along the slope of the eminence. The amount of contact between the implant and superior structures depends on the slope of the eminence, size and shape of the condyle, etc.

\section{Results}

The testing jig successfully applied a load/motion profile that simulated normal swine chewing. The average frequency of the mastication mechanism was $0.92 \mathrm{~Hz}( \pm 0.03 \mathrm{~Hz}$; Fig. 4). The compression phase had an average duration of $0.15 \mathrm{~s}( \pm 0.005 \mathrm{~s})$; the average peak compression force was $67.29 \mathrm{~N}( \pm 11.31 \mathrm{~N})$. The translational phase had an average duration of $0.428 \mathrm{~s}( \pm 0.045 \mathrm{~s})$; the average peak vertical force during the translational phase was $8.67 \mathrm{~N}( \pm 1.13 \mathrm{~N})$. The pauses between movements were an additional $0.51 \mathrm{~s}$ ( $\pm 0.071 \mathrm{~s})$.

\section{Discussion}

We developed a new bio-mimetic mechanical mastication mechanism based upon a biologic tissue environment. It was successfully used to perform wear testing of a novel temporomandibular joint implant. The magnitude and timing of the applied forces resemble data from previous in vivo $[14,18]$ and computational models [22]. The chewing frequency we recorded $(0.92 \mathrm{~Hz})$ approximates that of human mastication $[\sim 1 \mathrm{~Hz} ; 4]$. The average duration of the compression phase $(0.15 \mathrm{~s})$ closely resembles the computer model $[0.12-0.15 \mathrm{~s} ; 22]$ and swine in vivo measurements $[0.033-0.167 \mathrm{~s} ; 14]$. The average compression force $(67.29 \mathrm{~N})$ was similar to the computational model $[\sim 60 \mathrm{~N} ; 22]$. In vivo load- ing, like our mastication mechanism, demonstrates variability between compression forces in chewing cycles.

This mechanism is capable of re-creating the masticatory biomechanics of a variety of species because of its versatility. The air compression valves can be adjusted to produce larger or smaller compression forces. The chewing frequency of the mastication mechanism can be controlled by adjusting the pauses between each cycle and between the cycle's movements (compression then translation). Finally, the translational distances can be adjusted using the programmable logic controller, where any translation up to $50 \mathrm{~mm}$ can be accomplished.

The jig was stopped periodically through the testing to inspect the joint and implant surfaces for damage. The adjustment range of the vertical and horizontal linear actuators enabled quick and efficient exposure of the implant/joint. The rubber sac effectively enabled the joint to be submerged in a saline environment during the cyclic loading, but the sac could be adjusted to lower the fluid level below the height of the mandibular condyle in order to clearly view and photograph the implant and joint.

Our experimental tests utilized fully mature sow heads since several studies have reported that pigs are the best animal model for the human TMJ $[3,8$, 16]. Swine and humans have similar TMJ function which is the presumed reason why their anatomy is similar [16]. Fully mature specimens ensured complete development of the temporomandibular bones 
and thinner articular cartilage compared to immature specimens. If we had used immature specimens, with bones that were not fully calcified, then structural support beneath the implant may have been reduced which would not be relevant to the intended application in humans.

\subsection{Limitations}

The adjustment capacity of the compression forces and the translational distances represent a considerable advantage over other mechanisms. This mastication mechanism is capable of more accurately mimicking the in vivo biomechanics of various species; however, certain movements will directly relate to the maximum possible chewing frequency of the mechanism. For example, as the translational distance increases, the amount of time that is required in order to complete one chewing cycle increases. The compression phase has a short duration; accordingly it can occur at a higher frequency, closer to that of swine. The translations require more time since our linear actuator had a relatively low maximum velocity. Therefore, in our mechanism, the translations were the biggest factor limiting the chewing frequency to a maximum of $\sim 1.2 \mathrm{~Hz}$. This limited chewing frequency does not accurately match the swine chewing frequency $[\sim 3 \mathrm{~Hz} ; 18]$ and it also reduces the total number of chewing cycles in a given amount of time. The use of biologic tissues offers advantages because it incorporates the biological variability between TMJ's; however, the testing duration is limited due to degradation of the specimens. All in vitro studies with biological specimens test for a somewhat limited amount of time and therefore describe a rather limited number of loading cycles compared to in vivo joints.

Compressions and translations are the primary movements of the mandible. In swine, these movements have a rotational component [ $\sim 15-20$ degrees; 5] that allow the jaw to depress farther. In our mechanism the temporal portion translated perpendicular to the compression forces without any rotation. As a result, the mandibular condyles had a smaller contact area with the articular eminence and fossa than if they had a concurrent rotation. This mechanism was a preliminary step at bridging the gap between traditional synthetic implant testing and in vivo testing. Future work with this mechanism should focus around more accurately mimicking all the stages of masticatory biomechanics.

\subsection{Future work}

Our current testing approach represents a worst-case scenario (high loads and small contact area); however, future work with this mechanism should focus on adding a rotational component during mandibular depression and elevation movements. Rotations would increase the range of contact area between the implant and the articular eminence/articular fossa, resulting in a higher surface area abrasion. Further research with this mechanism will involve the addition of a rotational component, as it is an essential movement for the TMJ. In addition, incorporating actuators with higher linear velocities would enable higher chewing frequencies. This would give us the capability to mimic species with higher chewing frequencies, such as swine [18]. These changes would increase the biologic fidelity of the mastication mechanism and are important in the refinement of the apparatus. The ideal next step for testing would be to validate our mechanism by applying strain gauges on the condylar neck of the specimens and comparing the experimental data to published strain data from in vivo animals chewing [25].

\section{Conclusion}

This newly designed mastication mechanism is capable of applying chewing profiles to implants/joint specimens. The main advantage of this mechanism is the ability to adjust forces and times for both translational and compression movements. A secondary benefit includes the ability to incorporate biological variability. This mechanism design bridges the gap between traditional synthetic implant materials/mechanical testing and clinical trials. It will benefit the field of TMJ implantation the most if this mechanism and others like it are applied prior to in vivo research. The future of advanced clinical joint care will involve bio-mimetic solutions, such as this mechanism, that can properly test the efficacy of developing implants and accurately decrease the risks to the patient.

\section{Acknowledgments}

The authors thank Mr. Gary Morphy for his input on mechanism design and the fabrication of the novel meniscal replacement implants. This study was funded 
by the Ontario Centres of Excellence with partial support for the creation of the mastication mechanism from 4ibio Pickering, Ontario, Canada. All testing was completed at the Joint Biomechanics Laboratory at the University of Western Ontario, London, Ontario, Canada.

\section{References}

[1] J. Al-Sukhun, N. Ashammakhi and H. Penttila, Effects of tissue-engineered articular disc implants on the biomechanical loading of the human temporomandibular joint in a three-dimensional finite element model, J Craniofac Surg 18 (2007), 781-788.

[2] J. Al-Sukhun, J. Kelleway and M. Helenius, Development of a three-dimensional finite element model of a human mandible containing endosseous dental implants. I. Mathematical validation and experimental verification, J Biomed Mater Res 80 (2007), 234-246.

[3] A. Bermejo, O. Gonzalez and J.M. Gonzalez, The pig as an animal model for experimentation on the temporomandibular articular complex, Oral Surg Oral Med Oral Pathol 75 (1993), 18-23.

[4] R.L. Boyd, C.H. Gibbs, P.E. Mahan, A.F. Richmond and J.L. Laskin, Temporomandibular joint forces measured at the condyle of Macaca arctoides, Am J Orthod Dentofacial Orthop 97 (1990), 472-479.

[5] E.L. Brainerd, D.B. Baier, S.M. Gatesy, T.L. Hedrick, K.A. Metzger, S.L. Gilbert and J.J. Crisco, X-ray reconstruction of moving morphology (XROMM): Precision, accuracy and applications in comparative biomechanics research, $J$ Exp Zool A Ecol Genet Physiol 313 (2010), 262-279.

[6] K.H.S. Chan, T. Pearce, R.W. Blake, L. Chow, S. Wu, F. Wong and J. Li, Brief Communication: Simple mathematical and computations wear model for ultra-high-molecular-weight polyethylene total hip replacements, App Bion and Biomech 4 (2007), 83-88.

[7] R.J. De Kanter, G.J. Truin, R.C. Burgersdijk, M.A. Van 't Hof, P.G. Battistuzzi, H. Kalsbeek and A.F. Kayser, Prevalence in the Dutch adult population and a meta-analysis of signs and symptoms of temporomandibular disorder, J Dent Res 72 (1993), 1509-1518.

[8] M.S. Detamore, K.A. Athanasiou and J. Mao, A call to action for bioengineers and dental professionals: Directives for the future of TMJ bioengineering, Ann Biomed Eng 35 (2007), 1301-1311.

[9] O. Driemel, T. Ach, U.D. Muller-Richter, M. Behr, T.E. Reichert, M. Kunkel and R. Reich, Historical development of alloplastic temporomandibular joint replacement before 1945, Int J Oral Maxillofac Surg 38 (2009), 301-307.

[10] M.G. Fontenot and J.N. Kent, In vitro wear performance of Proplast TMJ disc implants, J Oral Maxillofac Surg 50 (1992), 133-139.

[11] J.R. Fricton, J.O. Look, E. Schiffman and J. Swift, Long-term study of temporomandibular joint surgery with alloplastic implants compared with nonimplant surgery and nonsurgical rehabilitation for painful temporomandibular joint disc displacement, J Oral Maxillofac Surg 60 (2002), 14001411 .
[12] C.H. Henry and L.M. Wolford, Treatment outcomes for temporomandibular joint reconstruction after Proplast-Teflon implant failure, Journal of Oral and Maxillofacial Surgery 51 (1993), 352-358.

[13] S. Hepguler, Y.S. Akkoc, M. Pehlivan, C. Ozturk, G. Celebi, A. Saracoglu and B. Ozpinar, The efficacy of intra-articular sodium hyaluronate in patients with reducing displaced disc of the temporomandibular joint, J Oral Rehabil 29 (2002), 80-86.

[14] S.W. Herring, The dynamics of mastication in pigs, Arch Oral Biol 21 (1976), 473-480.

[15] S.W. Herring, TMJ anatomy and animal models, J Musculoskelet Neuronal Interact 3 (2003), 391-394.

[16] S.W. Herring, J.D. Decker, Z.J. Liu and T. Ma, Temporomandibular joint in miniature pigs: Anatomy, cell replication, and relation to loading, Anat Rec 266 (2002), 152-166.

[17] S.W. Herring and Z.J. Liu, Loading of the temporomandibular joint: Anatomical and in vivo evidence from the bones, Cells Tissues Organs 169 (2001), 193-200.

[18] S.W. Herring and R.P. Scapino, Physiology of feeding in miniature pigs, J Morphol 141 (1973), 427-460.

[19] S. Ingawale and T. Goswami, Temporomandibular joint: Disorders, treatments, and biomechanics, Ann Biomed Eng 37 (2009), 976-996.

[20] A. Kashi, A.R. Chowdhury and S. Saha, Finite element analysis of a TMJ implant, J Dent Res 89 (2010), 241245.

[21] A. Kashi, S. Saha and R.W. Christensen, Temporomandibular joint disorders: Artificial joint replacements and future research needs, J Long-Term Eff Med Implants 16 (2006), 459-474.

[22] G.E. Langenbach, F. Zhang, S.W. Herring and A.G. Hannam, Modelling the masticatory biomechanics of a pig, J Anat 201 (2002), 383-393.

[23] Laskin DM, Internal Derangements, In: TMD's an evidencebased approach to Diagnosis and Treatment, Quintessence Publishing Co., Inc., Hanover Park, Illinois, 2006, pp. 249253.

[24] A.L. Lippincott III, J.M. Dowling, D. Phil, J.B. Medley and R.W. Christensen, Temporomandibular Joint Arthroplasty Using Metal-on-Metal and Acrylic-on-Metal Configurations Wear in Laboratory Tests and In Retrievals, Surg Technol Int 8 (2000), 321-330.

[25] L. Marks, S. Teng, J. Artun and S. Herring, Reaction strains on the condylar neck during mastication and maximum muscle stimulation in different condylar positions: An experimental study in the miniature pig, J Dent Res 76 (1997), 14121420 .

[26] L.G. Mercuri, The use of alloplastic prostheses for temporomandibular joint reconstruction, J Oral Maxillofac Surg 58 (2000), 70-75

[27] M.J. Papo, S.A. Catledge, Y.K. Vohra and C. Machado, Mechanical wear behavior of nanocrystalline and multilayer diamond coatings on temporomandibular joint implants, $J$ Mater Sci Mater Med 15 (2004), 773-777.

[28] O.C. Rasmussen, Description of population and progress of symptoms in a longitudinal study of temporomandibular arthropathy, Scand J Dent Res 89 (1981), 196203.

[29] D.E.T. Shepherd and G. Azangwe, Synthetic versus tissueengineered implants for joint replacement, App Bion and Biomech 4 (2007), 179-185. 
[30] B.J. Sindelar and S.W. Herring, Soft tissue mechanics of the temporomandibular joint, Cells Tissues Organs 180 (2005), $36-43$.

[31] B. Stegenga, L.G. de Bont and G. Boering, Osteoarthrosis as the cause of craniomandibular pain and dysfunction: A unifying concept, J Oral Maxillofac Surg 47 (1989), 249-256.
[32] P.A. Toller, Temporomandibular capsular rearrangement, $\mathrm{Br}$ J Oral Surg 11 (1974), 207-212.

[33] J.P. van Loon, G.J. Verkerke, M.P. de Vries and L.G. de Bont, Design and wear testing of a temporomandibular joint prosthesis articulation, J Dent Res 79 (2000), 715-721. 

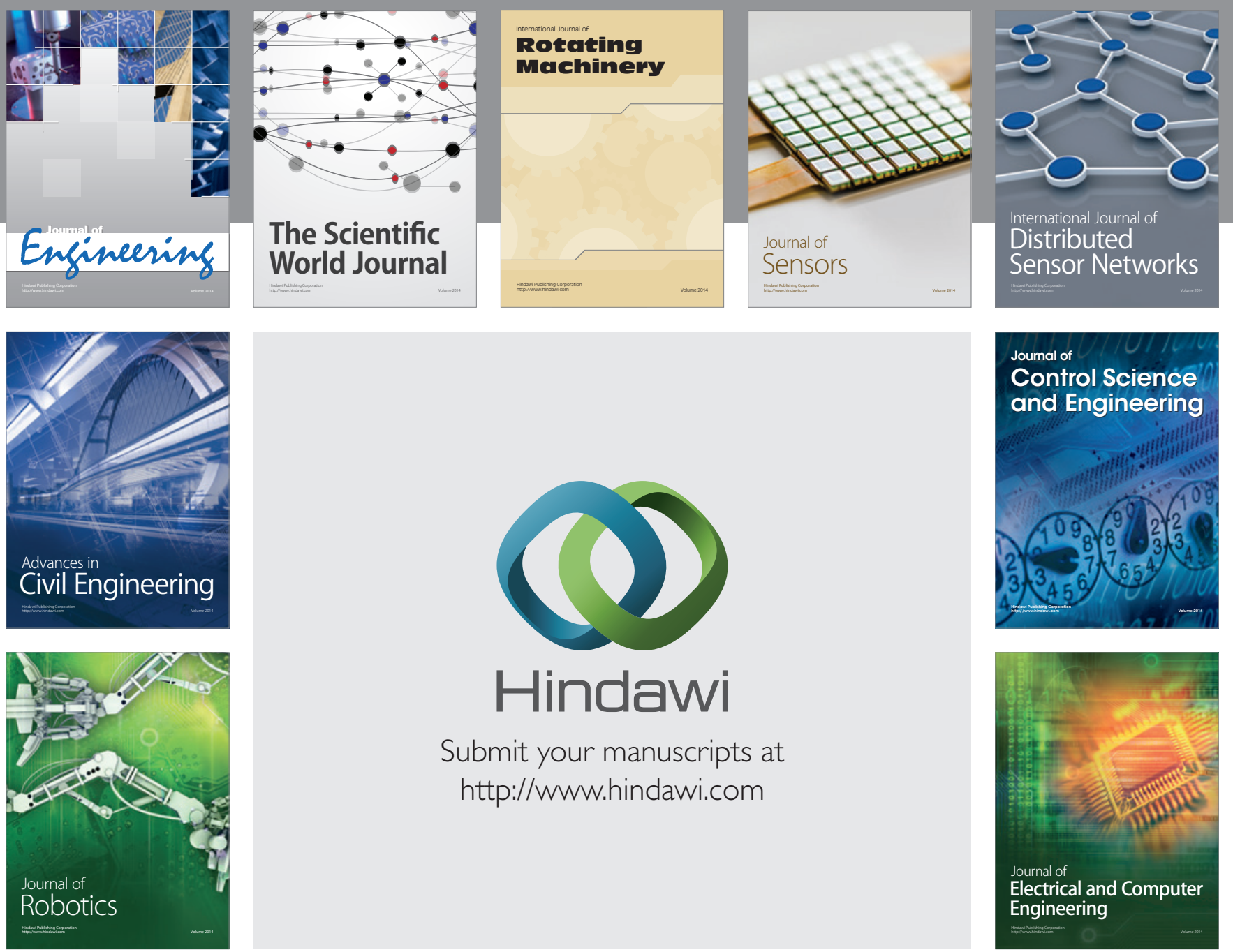

Submit your manuscripts at

http://www.hindawi.com
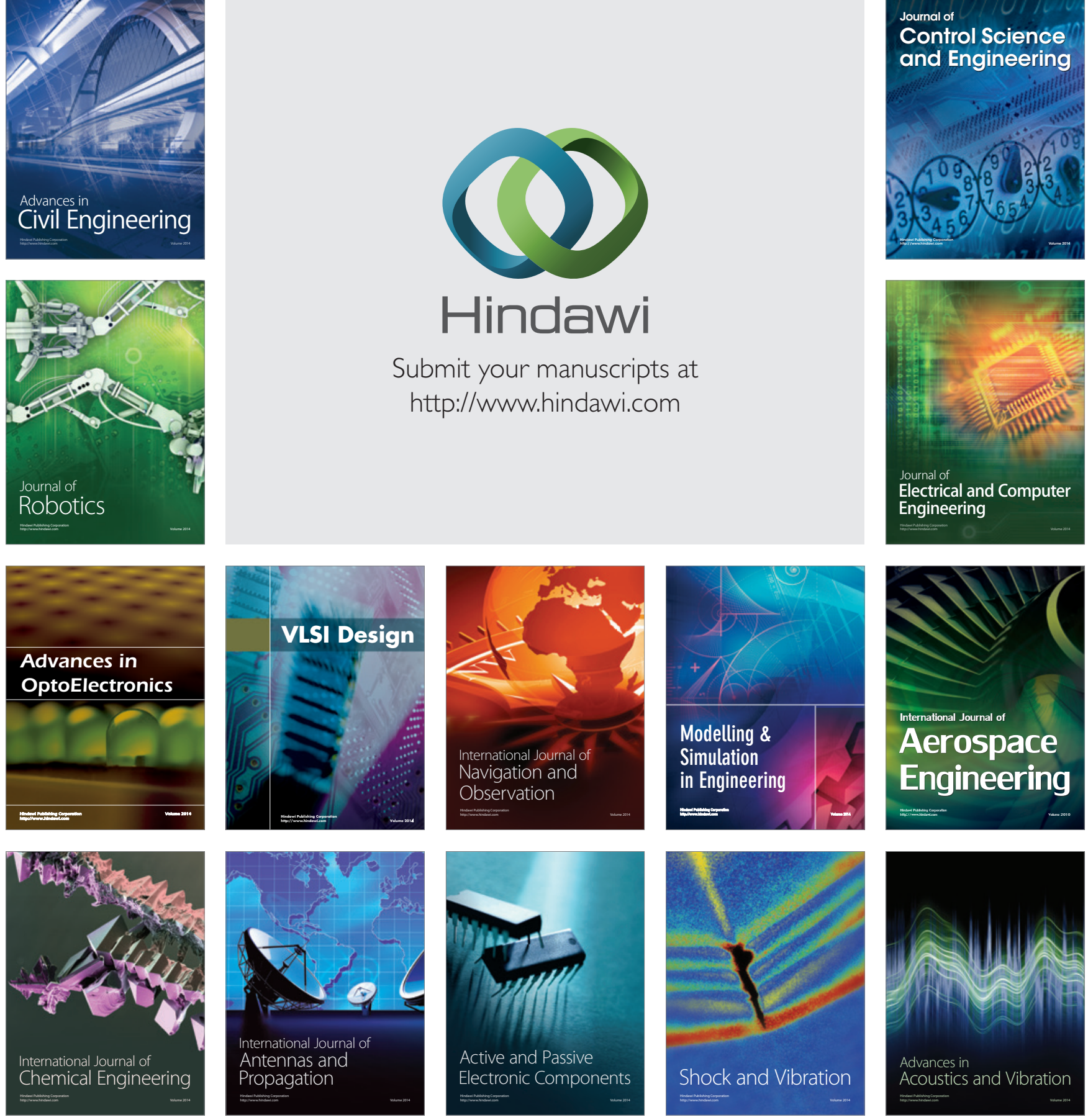